\title{
Identity-based conditional proxy re-encryption with fine grain policy
}

\author{
Chunpeng $\mathrm{Ge}^{1}$, Willy Susilo ${ }^{2 *}$ Jiandong Wang ${ }^{3} \&$ Liming Fang ${ }^{3}$ \\ ${ }^{1}$ Department of Computer Engineering, \\ Jiangsu University of Technology, Changzhou, China \\ Email: gecp@nuaa.edu.au \\ ${ }^{2}$ Centre for Computer and Information Security Research (CCISR), \\ School of Computing and Information Technology, \\ University of Wollongong, Australia \\ Email: wsusilo@uow.edu.au \\ ${ }^{3}$ College of Computer Science and Technology \\ Nanjing University of Aeronautics and Astronautics, Nanjing, China
}

\begin{abstract}
An identity-based conditional proxy re-encryption scheme (IB-CPRE) allows a semi-trusted proxy to convert a ciphertext satisfying one condition, which is set by the delegator, under one identity to another without the necessity to reveal the underlying message. In ICISC 2012, Liang, Liu, Tan, Wong and Tang proposed an IB-CPRE scheme, and left an open problem on how to construct chosen-ciphertext secure IB-CPRE supporting OR gates on conditions. In this work, we answer this aforementioned problem by constructing an identitybased conditional proxy re-encryption scheme with fine grain policy (IB-CPRE-FG). In an IB-CPRE-FG scheme, each ciphertext is labeled with a set of descriptive conditions and each re-encryption key is associated with an access tree that specifies which type of ciphertexts the proxy can re-encrypt. Furthermore, our scheme can be proved secure against adaptive access tree and adaptive identity chosen-ciphertext attack.
\end{abstract}

Keywords: Conditional proxy re-encryption, Identity-based proxy re-encryption, Chosenciphertext security.

\section{Introduction}

Identity-based proxy re-encryption(IB-PRE), introduced by Green and Ateniese [1], enables a proxy to convert a ciphertext encrypted under Alice's identity into one encrypted under Bob's identity. To capture the conditional property, Shao, Wei, Ling and Xie [2] introduced the notion of identity-based conditional proxy re-encryption(IB-CPRE), in which only ciphertexts satisfying a special condition can be convert by the proxy. However, their scheme is only proven secure in the random oracle. Further, Liang, Liu, Tan, Wong and Tang [3] proposed an identity-based conditional proxy re-encryption scheme in the standard model.

${ }^{*}$ Corresponding Author: Willy Susilo. 
Unfortunately, the above scheme [3] does not allow any boolean combinations of conditions. However, boolean combinations of conditions are essential practical property for CPRE. We shall use the on-line medical service systems [4] to illustrate the practicality of our work. In an on-line medical system, a patient prefers to find doctors with the following requirements to remedy his heart disease. The requirements are described by a set of attributes $W=$ ("heart", "protomedicus", "specialist", "New York"). The patient encrypts his medical record with $W$ before uploading to the system. The system then forwards the ciphertext to doctor Alice to in charge of this case. Nevertheless, when the doctor is out for a vacation, he may want to find a trustworthy colleague Bob with policy $P_{1}=$ ("heart" $\bigwedge($ "protomedicus" $\bigvee$ "specialist") $)$ (Location: "Downtown of New York") to check the medical record for him. In a traditional public key encryption system, Alice has to decrypt the ciphertext and recover the date first, and then encrypt the date using Bob's public key. If there are $N$ different patients' medical records that Alice wants to send to $N$ different colleagues, she is required to perform $N$ decryptions and $N$ new encryptions. This is impractical as Alice's workload is linear in $N$. The situation becomes worse as she should be on-line during each encryption process.

To solve the above problem, many CPRE schemes were proposed to capture a fine-grained control over conditions $[5,6,7]$. However, none of the these schemes work in the identitybased setting. In 2011, Fang, Susilo, Ge and Wang proposed an interactive conditional proxy re-encryption with fine grain policy [8] and left an open problem on how to construct a non-interactive conditional proxy re-encryption with fine grain policy.

\subsection{Our contribution}

This work, for the first time, answers the aforementioned open problems affirmatively by presenting an identity-based proxy re-encryption with fine grain policy. Our contributions are as follows. First, we propose a fine grain policy on conditions. In this work, the access control on conditions is described an access tree. Hence, our work moves one step ahead to solve the open question proposed by [3]. Finally, as our condition proxy re-encryption works in the identity-based setting, and the re-encryption key is generated without any interaction between the delegator and the delegatee, our scheme is also an affirmative answer of the open question proposed by [8]. Moreover, Our scheme is secure against adaptive access tree and adaptive identity chosen-ciphertext attack under the decisional bilinear Diffie-Hellman assumption.

\subsection{Related work}

Proxy re-encryption. Proxy re-encryption(PRE) was first introduced by Blaze, Bleumer and Strauss [9], which enables a semi-trusted proxy to transform Alice's ciphertext into Bob's ciphertext of the same message. The proxy, however, cannot learn anything about the underlying message. In 2005, the first unidirectional PRE scheme was proposed by Ateniese, Fu, Green and Hohenberger [10]. Follow their work, many PRE schemes [11, 12, 13, 14, 15] with different properties have been proposed. To make flexible control on encrypted ciphertexts, conditional proxy re-encryption(CPRE) schemes $[16,17,8,18]$ were proposed, in which only ciphertexts satisfying a certain condition can be re-encrypted.

Identity-based proxy re-encryption. To extend the notion of proxy re-encryption to the identity-based setting, Green and Ateniese [1] proposed the first identity-based proxy 
re-encryption (IB-PRE). In an IB-PRE scheme, the proxy can convert a ciphertext encrypted under Alice's identity into one encrypted under Bob's identity. They proposed two IB-PRE schemes, which are both secure in the random oracle model. Chun and Tzeng [19] introduced two IB-PRE schemes, which are both secure in the standard model based on Waters IBE scheme [20]. However, in previous schemes, the length of re-encrypted ciphertext grows with the time of re-encryption. To solve this problem, Liang et al. [21] proposed a CCAsecure multi-hop identity-based conditional proxy re-encryption scheme with constant-size ciphertexs. Liang, Susilo and Liu [22] proposed an anonymous multi-hop identity-based conditional proxy re-encryption scheme and found it can provide privacy-preserving for big data storage. In ASIACCS 2009, Liang et al. [5] introduced the notion of attribute-based proxy re-encryption(AB-PRE), in which identities are viewed as a set of attribute. To support AB-PRE with any monotonic access structures, ciphertext-policy AB-PRE and key-policy AB-PRE were proposed by Liang et al. [23, 24, 25, 26] and Ge et al. [27]. In 2014, Liang et al. [28] introduced a new notion of DFA-based functional PRE and proposed a concrete DFA-based functional PRE scheme with CCA security.

Lattice-based proxy re-encryption. The vast majority of schemes based on number theoretic problems such as the discrete logarithm are known to be susceptible against quantum computers attack. To improve this problem, Aono et al. [29] proposed an unidirectional proxy re-encryption scheme under LWE. Their scheme is CPA-secure in the standard model and can be transformed into CCA-secure in the random oracle. After their work, Kirshanova [30] proposed a CCA1 secure proxy re-encryption scheme in the selective model based on LWE. In ASIACCS 2015, Nunez, Agudo and Lopez [31] proposed bidirectional and multi-hop proxy re-encryption based on NTRU.

\section{$1.3 \quad$ Roadmap}

The rest of the paper is organized as follows. We first provide the basic primitives and the security model for an IB-CPRE-FG scheme in Sec. 2. In Sec. 3, we present our IB-PRE-FG scheme and give the security proofs. We give a comparison and discussion of our scheme in Sec. 4. Finally, we conclude the paper in Sec. 5 .

\section{Preliminaries}

\section{$2.1 \quad$ Negligible function}

A function $\varepsilon(n): N \rightarrow R$ is said to be negligible if for all positive integer $c \in N$ there exists a $n_{c} \in N$ such that $\varepsilon(n)<n_{c}$ for all $n>n_{c}$.

\subsection{Bilinear map}

Let $G$ and $G_{T}$ be two multiplicative cyclic groups with the same prime order $p$, and $g$ be a generator of $G$. A bilinear pairing is a map $e: G \times G \rightarrow G_{T}$ with the following properties $[32,33]$ :

1. $e\left(g^{a}, h^{b}\right)=e(g, h)^{a b}$ for all $a, b \stackrel{R}{\leftarrow} Z_{p}^{*}$ and $g, h \in G$.

2. $e(g, g) \neq 1$. 
3. There is an efficient algorithm to compute $e(g, h)$ for all $g, h \in G$.

\subsection{Decisional bilinear Diffie-Hellman(DBDH) assumption}

The DBDH assumption [20] is as below: Given a vector of 5 elements

$$
\left(g, g^{a}, g^{b}, g^{c}, T\right) \in G^{4} \times G_{T}
$$

as input, it is hard to distinguish $T=e(g, g)^{a b c}$ from a random value in $G_{T}$. Formally, for all probability polynomial time adversaries $\mathcal{A}$, the following probability is negligible:

$$
\begin{aligned}
& \mid \operatorname{Pr}\left[a, b, c, r \stackrel{R}{\longleftarrow} Z_{p}^{*} ; \quad T_{0}=e(g, g)^{a b c} ; \quad T_{1}=e(g, g)^{r} ;\right. \\
& \left.z \in\{0,1\} ; \quad z^{\prime} \leftarrow \mathcal{A}\left(g, g^{a}, g^{b}, g^{c}, T_{z}\right): \quad z=z^{\prime}\right] \mid-\frac{1}{2} .
\end{aligned}
$$

\subsection{Strongly unforgeable one-time signature}

A one-time signature [34] consists of a triple of algorithms $\operatorname{Sig}=(\mathcal{G}, \mathcal{S}, \mathcal{V})$ such that, on input of a security parameter $\lambda, \mathcal{G}$ generates a one-time key pair $(s s k, s v k)$, while for any message $M, \mathcal{V}(s v k, \sigma, M)$ outputs 1 whenever $\sigma=\mathcal{S}(s s k, M)$ and 0 otherwise. As in [11], we need strongly unforgeable one-time signatures, which means that no PPT adversary can create a new signature even for a previously signed message.

$\operatorname{Sig}=(\mathcal{G}, \mathcal{S}, \mathcal{V})$ is a strongly unforgeable one-time signature if the probability $A d v^{O T S}=$ $\operatorname{Pr}\left[(s s k, s v k) \leftarrow G(\lambda) ;(M, S t) \leftarrow F(s v k) ; \sigma \leftarrow \mathcal{S}(s s k, M) ;\left(M^{\prime}, \sigma^{\prime}\right) \leftarrow F(M, \sigma, s v k, S t):\right.$ $\left.\mathcal{V}\left(s v k, \sigma^{\prime}, M^{\prime}\right)=1 \wedge\left(M^{\prime}, \sigma^{\prime}\right) \neq(M, \sigma)\right]$, where $S t$ denotes the state information maintained by $F$ between stages, is negligible for any PPT forger $F$.

\subsection{Pseudorandom function family}

We here review the definition of pseudorandom function family [13]. Let $F: \mathcal{K} \times \mathcal{D} \rightarrow \mathcal{R}$ be a function family, where $\mathrm{K}$ is the set of keys of $F, D$ is the domain, and $R$ is the range. Let $G: D \rightarrow R$ be a true random function family. Let $\mathcal{F}$ be a PPT adversary which outputs a bit. We consider the following two experiments:

$$
\begin{gathered}
\text { Experiment } \operatorname{Exp}_{F}^{P R F-1}(\mathcal{F}):\left[K \stackrel{\$}{\leftarrow} \mathcal{K}, b \stackrel{\$}{\leftarrow} \mathcal{F}^{\mathcal{F}(\mathcal{K}, .)}, \text { Return } b\right] \\
\text { Experiment } \operatorname{Exp}_{F}^{P R F-0}(\mathcal{F}):\left[g \stackrel{\$}{\leftarrow} G, b \stackrel{\$}{\leftarrow} \mathcal{F}^{\mathcal{G}(.)}, \text { Return } b\right] .
\end{gathered}
$$

We define $\mathcal{F}$ 's advantage $A d v_{F, \mathcal{F}}^{P} R F$ in attacking the pseudorandom of the function $F$ as

$$
\left|\operatorname{Pr}\left[\operatorname{Exp}_{F}^{P R F-1}(\mathcal{F})=1\right]-\operatorname{Pr}\left[\operatorname{Exp}_{F}^{P R F-0}(\mathcal{F})=1\right]\right|
$$

If for any PPT adversary $\mathcal{F}$, its advantage in attacking the pseudorandomness of the function family $F$ is negligible, the we say that $F$ is a pesudorandom function family. 


\subsection{Identity-based proxy re-encryption with fine grain policy}

In this subsection, we will provide the definition of identity-based proxy re-encryption with fine grain policy. We use the same approach as [8], where ciphertexts are labeled with a set of descriptive conditions. A tree access structure, in which each interior node of the tree is a threshold gate and the leaves are associated with conditions, is used to identify re-encryption keys. Using this approach, we can present a tree with "AND" and "OR" gate by using $n$-outof- $n$ and 1-out-of- $n$ threshold gates, respectively. A user will be able to re-encrypt a ciphertext with a re-encryption key provided if and only if there is an assignment of conditions from the ciphertexts to nodes of the tree such that the tree is satisfied.

Definition 1 (Access tree [35]). Access tree $\mathcal{T}$. We incorporate the definition of access tree as in [35]. Let $\mathcal{T}$ be a tree that represents an access structure. Each non-leaf node of the tree represents a threshold gate, described by its children a threshold value. Let num $_{x}$ be the number of children of a node $x$ and $k_{x}$ be its threshold value, then we have $0<k_{x} \leq n u m_{x}$. When $k_{x}=$ num $_{x}$, it is an AND gate, and when $k_{x}=1$, the threshold is an OR gate. Each leaf node $x$ of the tree is described by a conditional keyword and a threshold value $k_{x}=1$. We fit the root of an access tree to be at depth 0 . Let $\overline{L N_{\mathcal{T}}}$ be the set of all the non-leaf nodes, and $L N_{\mathcal{T}}$ be the set of all the leaf nodes. We denote the parent of node $x$ in the tree $p(x)$. The function keyword $(x)$ is defined only if $x \in L N_{\mathcal{T}}$ and it denotes the conditional keyword associated with the leaf node $x$ in the tree. The access tree $\mathcal{T}$ also defines an ordering between the children of every node, that is, the children of a node are numbered from 1 to num. The function inex $(z)$ returns a index associated with the node $z$, where the index values are uniquely assigned to nodes in the access tree.

Satisfying an access tree. Let $\mathcal{T}$ be an access tree with root $r . \mathcal{T}_{x}$ is the subtree rooted at the node $x$. Hence $\mathcal{T}$ is the same as $\mathcal{T}_{r} . \mathcal{T}_{x}(W)=1$ denotes a set of conditions $W$ satisfies the access tree $\mathcal{T}_{x}$. If $x \in L N_{\mathcal{T}}$, then $\mathcal{T}_{x}(W)=1$ if and only if $\operatorname{keyword}(x) \in W$. If $x \in \overline{L N_{\mathcal{T}}}$, evaluate $\mathcal{T}_{z}(W)$ for all children $z$ of node $x, \mathcal{T}_{x}(W)$ return 1 if and only if at least $k_{x}$ children return 1 .

Definition 2 (IB-CPRE-FG). An (single-use) identity-based conditional proxy re-encryption with fine grain policy [3] scheme consists the following algorithms:

1. $\operatorname{Setup}(\lambda)$ : The Setup algorithm is run by the private key generator(PKG), on input a security parameter $\lambda$, the global public parameters $P P$ and mast secret key $m s k$ are outputted.

2. KeyGen $(m s k, I D)$ : On input the mast secret key msk and an identity $I D$, output the private key $s k_{I D}$ for identity $I D$.

3. $\operatorname{Enc}(I D, m, W)$ : On input an identity $I D$, a message $m \in M$ and a condition set $\mathrm{W}$, output a second level ciphertext $C_{I D}^{(2)}$. Here $M$ denotes the message space.

4. RKeyGen $\left(s k_{I D_{i}}, I D_{i}, \mathcal{T}, I D_{j}\right)$ : On input identities $I D_{i}, I D_{j}$, the secret key $s k_{I D_{i}}$ and an access tree $\mathcal{T}$, output the re-encryption key $r k_{\mathcal{T} \mid I D_{i} \rightarrow I D_{j}}$.

5. ReEncrypt $\left(C_{I D_{i}}^{(2)}, r k_{\mathcal{T} \mid I D_{i} \rightarrow I D_{j}}\right)$ : On input a re-encryption key $r k_{\mathcal{T} \mid I D_{i} \rightarrow I D_{j}}$ and a second level ciphertext $C_{I D_{i}}^{(2)}$ associated with $\mathrm{W}$ under identity $I D_{i}$, output a first level ciphertext $C_{I D_{j}}^{(1)}$. 
6. Dec2 $\left(I D_{i}, s k_{I D_{i}}, C_{I D_{i}}^{(2)}\right)$ : On input an identity $I D_{i}$, a private key $s k_{I D_{i}}$, and a second level ciphertext $C_{I D_{i}}^{(2)}$, output the plaintext $m$ or an error symbol $\perp$.

7. $\operatorname{Dec} 1\left(I D_{j}, s k_{I D_{j}}, C_{I D_{j}}^{(1)}\right)$ : On input an identity $I D_{j}$, a private key $s k_{I D_{j}}$, and a first level ciphertext $C_{I D_{j}}^{(1)}$, output the plaintext $m$ or an error symbol $\perp$.

Note that we omit the global parameters $P P$ as the other algorithms' input for simplicity. The correctness of IB-CPRE-FG means that, for any message $m \in M, s k_{I D_{i}} \leftarrow$ $\operatorname{KeyGen}\left(m s k, I D_{i}\right), s k_{I D_{j}} \leftarrow \operatorname{KeyGen}\left(m s k, I D_{j}\right)$, and $r k_{\mathcal{T} \mid I D_{i} \rightarrow I D_{j}} \leftarrow \operatorname{RKeyGen}\left(s k_{I D_{i}}, I D_{i}\right.$, $\left.\mathcal{T}, I D_{j}\right)$ and $C_{I D_{i}}^{(2)}=\left(I D_{i}, m, W\right)$, where $\mathcal{T}(W)=1$.

$$
\operatorname{Pr}\left[\operatorname{Decrypt}\left(C_{I D_{i}}^{(2)}, s k_{I D_{i}}\right)=m\right]=1
$$

and

$$
\operatorname{Pr}\left[\operatorname{Decrypt}\left(\operatorname{sk}_{I D_{j}}, \operatorname{ReEncrypt}\left(C_{I D_{i}}^{(2)}, r k_{\mathcal{T} \mid I D_{i} \rightarrow I D_{j}}\right)\right)=m\right]=1 .
$$

\subsection{Security notion for IB-CPRE-FG}

Next, we will provide the game-based security definition of IB-CPRE-FG. As in [3], our security model considers the indistinguishability of the second level ciphertext and the first level ciphertext in the adaptive identity and adaptive condition set model. The two security game are denoted as IND-level2-CCA and IND-level1-CCA individually.

Definition 3 (IND-level2-CCA). An (single-use) IB-CPRE-FG scheme is IND-level2-CCA secure if no probabilistic polynomial time(PPT) adversary $\mathcal{A}$ can win the game below with non-negligible advantage. Next in the game, assuming $\mathcal{B}$ is the challenger and $\lambda$ is the security parameter.

1. Setup. $\mathcal{B}$ Runs the $\operatorname{Setup}(\lambda)$ algorithm to get the (PP,msk), and give PP to $\mathcal{A}$.

2. Query phase 1. $\mathcal{A}$ makes the following queries:

(a) Extract $(I D)$ : run the KeyGen $(m s k, I D)$ algorithm to get $s k_{I D}$, and returns $s k_{I D}$ to $\mathcal{A}$.

(b) RKExtract $\left(I D_{i}, \mathcal{T}, I D_{j}\right)$ : run the $R K \operatorname{eyGen}\left(s k_{I D_{i}}, I D_{i}, \mathcal{T}, I D_{j}\right)$ algorithm to get $r k_{\mathcal{T} \mid I D_{i} \rightarrow I D_{j}}$, and returns $r k_{\mathcal{T} \mid I D_{i} \rightarrow I D_{j}}$ to $\mathcal{A}$.

(c) $\operatorname{ReEnc}\left(I D_{i}, I D_{j},\left(W, C_{I D_{i}}^{(2)}\right)\right)$ : run the $\operatorname{ReEncrypt}\left(C_{I D_{i}}^{(2)}, r k_{\mathcal{T} \mid I D_{i} \rightarrow I D_{j}}\right)$, where $\mathcal{T}(W)=$ 1 , algorithm to get a first level ciphertext $C_{I D_{j}}^{(1)}$, and returns $C_{I D_{j}}^{(1)}$ to $\mathcal{A}$.

(d) $\operatorname{Dec} 2\left(I D_{i},\left(W, C_{I D_{i}}^{(2)}\right)\right)$ : run the $\operatorname{Dec} 2\left(I D_{i}, s k_{I D_{i}}, C_{I D_{i}}^{(2)}\right)$ algorithm to get the underlying message $m$, and returns $m$ to $\mathcal{A}$.

(e) $\operatorname{Dec} 1\left(I D_{j}, C_{I D_{j}}^{(1)}\right)$ : run the $\operatorname{Dec} 1\left(I D_{j}, s k_{I D_{j}}, C_{I D_{j}}^{(1)}\right)$ algorithm to get the underlying message $m$, and returns $m$ to $\mathcal{A}$.

3. Challenge. Once $\mathcal{A}$ decides that Phase 1 is over, it outputs two equal length message $\left(m_{0}, m_{1}\right)$, a target identity $I D^{*}$ and a target condition set $W^{*}$. The challenger $\mathcal{B}$ chooses a random bit $b \in\{0,1\}$ and sends the challenge ciphertext $C_{I D^{*}}^{(2) *}=\operatorname{Enc}\left(I D^{*}, m_{b}, W^{*}\right)$ to $\mathcal{A}$, if the following queries are never made:

- Extract $\left(I D^{*}\right)$, and

- RKExtract $\left(I D^{*}, \mathcal{T}, I D_{j}\right)$ and $\operatorname{Extract}\left(I D_{j}\right)$, where $\mathcal{T}\left(W^{*}\right)=1$. 
4. Query phase 2. $\mathcal{A}$ continues making queries as in the Query phase 1, except the following queries:

(a) $\operatorname{Extract}\left(I D^{*}\right)$;

(b) RKExtract $\left(I D^{*}, \mathcal{T}, I D_{j}\right)$ and $\operatorname{Extract}\left(I D_{j}\right)$, where $\mathcal{T}\left(W^{*}\right)=1$;

(c) $\operatorname{ReEnc}\left(I D^{*}, I D_{j},\left(W^{*}, C_{I D^{*}}^{(2)}\right)\right)$ and $\operatorname{Extract}\left(I D_{j}\right)$;

(d) $\operatorname{Dec} 2\left(I D^{*},\left(W^{*}, C_{I D^{*}}^{(2) *}\right)\right)$;

(e) $\operatorname{Dec} 1\left(I D_{j}, C_{I D_{j}}^{(1)}\right)$, if $C_{I D_{j}}^{(1)}$ is a derivative of $C_{I D^{*}}^{(2) *}$. As defined in [11], derivative of $C_{I D_{i}}^{(2)}=\operatorname{Enc}\left(I D_{i}, m, W\right)$ is defined as follows:

$-C_{I D_{i}}^{(2)}$ is a derivative of itself;

- If $\mathcal{A}$ has issued $R K \operatorname{Extract}\left(I D_{i}, \mathcal{T}, I D_{j}\right)$ query to obtain a re-encryption key $r k_{\mathcal{T} \mid I D_{i} \rightarrow I D_{j}}$, where $\mathcal{T}(W)=1$, and computed $C_{I D_{j}}^{(1)} \rightarrow \operatorname{Re} \operatorname{Enc}\left(I D_{i}, I D_{j},\left(W, C_{I D_{i}}^{(2)}\right)\right)$, then $C_{I D_{j}}^{(1)}$ is a derivative of $C_{I D_{i}}^{(2)}$.

- If $\mathcal{A}$ has issued $\operatorname{Re} \operatorname{Enc}\left(I D_{i}, I D_{j},\left(W, C_{I D_{i}}^{(2)}\right)\right)$ and obtained $C_{I D_{j}}^{(1)}$. Then $C_{I D_{j}}^{(1)}$ is a derivative of $C_{I D_{i}}^{(2)}$.

5. Guess. $\mathcal{A}$ outputs the guess $b^{\prime}$. The adversary wins if $b^{\prime}=b$.

We say that an IB-CPRE-FG scheme is IND-level2-CCA secure, if the following probability is negligible for all probabilistic polynomial time adversary $\mathcal{A}$ :

$$
A d v_{\mathcal{A}}^{I N D-l e v e l 2-C C A}(\lambda)=\left|\operatorname{Pr}\left[b^{\prime}=b\right]-1 / 2\right| .
$$

Next, we will present the indistinguishability of the first level ciphertext. In this definition, a first level ciphertext is provided to the adversary $\mathcal{A}$ in the challenge phase. As defined in [12], since the first level ciphertext cannot be re-encrypted, $\mathcal{A}$ should be allowed to obtain any re-encryption key including those from the target identity to identities that the adversary have issued Extract query. Furthermore, sice $\mathcal{A}$ is allowed to obtain any re-encryption keys, the ReEnc query becomes useless. Moreover, the Dec2 query is also unnecessary.

Definition 4 (IND-level1-CCA). An (single-use) IB-CPRE-FG scheme is IND-level1-CCA secure if no probabilistic polynomial time(PPT) adversary $\mathcal{A}$ can win the game below with non-negligible advantage. Next in the game, assuming $\mathcal{B}$ is the challenger and $\lambda$ is the security parameter.

1. Setup. $\mathcal{B}$ Runs the $\operatorname{Setup}(\lambda)$ algorithm to get the $(\mathrm{PP}, \mathrm{msk})$, and give $\mathrm{PP}$ to $\mathcal{A}$.

2. Query phase 1. $\mathcal{A}$ makes the following queries:

(a) Extract $(I D)$ : run the $\operatorname{KeyGen}(m s k, I D)$ algorithm to get $s k_{I D}$, and returns $s k_{I D}$ to $\mathcal{A}$.

(b) RKExtract $\left(I D_{i}, \mathcal{T}, I D_{j}\right)$ : run the $R K \operatorname{eyGen}\left(s k_{I D_{i}}, I D_{i}, \mathcal{T}, I D_{j}\right)$ algorithm to get $r k_{\mathcal{T} \mid I D_{i} \rightarrow I D_{j}}$, and returns $r k_{\mathcal{T} \mid I D_{i} \rightarrow I D_{j}}$ to $\mathcal{A}$.

(c) $\operatorname{Dec} 1\left(I D_{j}, C_{I D_{j}}^{(1)}\right)$ : run the $\operatorname{Dec} 1\left(I D_{j}, s k_{I D_{j}}, C_{I D_{j}}^{(1)}\right)$ algorithm to get the underlying message $m$, and returns $m$ to $\mathcal{A}$. 
3. Challenge. Once $\mathcal{A}$ decides that Phase 1 is over, it outputs two equal length message $\left(m_{0}, m_{1}\right)$ and a target identity $I D^{*}$. The challenger $\mathcal{B}$ chooses a random bit $b \in\{0,1\}$ and sends the challenge ciphertext $C_{I D^{*}}^{(1) *}=\operatorname{Re} \operatorname{Enc}\left(\operatorname{Enc} 2\left(I D, m_{b}, W\right), r k_{\mathcal{T} \mid I D \rightarrow I D^{*}}\right)$ to $\mathcal{A}$, where $I D \neq I D^{*}$ and $\mathcal{T}(W)=1$. The restriction is that the adversary has never make the Extract $\left(I D^{*}\right)$ query.

4. Query phase 2. $\mathcal{A}$ continues making queries as in the Query phase 1, except the following queries:

(a) $\operatorname{Extract}\left(I D^{*}\right)$;

(b) $\operatorname{Dec} 1\left(C_{I D^{*}}^{(1) *}\right)$.

5. Guess. $\mathcal{A}$ outputs the guess $b^{\prime}$. The adversary wins if $b^{\prime}=b$.

We say that an IB-CPRE-FG scheme is IND-level1-CCA secure, if the following probability is negligible for all probabilistic polynomial time adversary $\mathcal{A}$ :

$$
A d v_{\mathcal{A}}^{I N D-\text { level } 1-C C A}(\lambda)=\left|\operatorname{Pr}\left[b^{\prime}=b\right]-1 / 2\right| .
$$

The IB-CPRE-FG scheme is said to be IND-CCA secure if both $A d v_{\mathcal{A}}^{I N D-\text { level2-CCA }}(\lambda)$ and $A d v_{\mathcal{A}}^{I N D-\text { level1-CCA }}(\lambda)$ are negligible.

\section{Our proposed IB-CPRE-FG}

In this section, we first propose our IB-CPRE-FG scheme and then prove its IND-CCA security.

\subsection{On achieving IND-CCA secure IB-CPRE-FG}

Inspired by [35], in our scheme, the ciphertext is encrypted with a condition set $W$ and the re-encryption key is generated with an access tree $\mathcal{T}$. The proxy is able to re-encrypt the ciphertext if and only if $\mathcal{T}(W)=1$. One may thought that it is trivial to achieve an IB-CPRE-FG scheme by combining an identity-based encryption and an attribute-based encryption(ABE). As in [8], one may use the the ABE scheme with the mast key $x_{i}$, and then subsequently alter the input of the attribute-key generation algorithm form $x_{i}$ to $x_{j}-x_{i}$. The re-encryption key $x_{j}-x_{i}$ is then involved in the access tree. Unfortunately, this approach can not be used directly in the identity-based setting. In the former scheme, the secret and public key for an user $i$ is set to be $x_{i}$ and $g^{x_{i}}$, where $x_{i} \in Z_{p}^{*}$ and $g^{x_{i}} \in G$. The re-encryption key $s k_{j}-s k_{i}=x_{j}-x_{i} \in Z_{p}^{*}$ is set to be the exponent for element $g^{x_{j}-x_{i}}$. However, in the identity-based setting [20,33], the secret key for an identity is set be an element in $G$ and the public key is $I D$. The re-encryption key $s k_{j}-s k_{i}$ cannot be used as the exponent anymore. Furthermore, the re-encryption key $s k_{j}-s k_{i}$ cannot be involved in the access tree using the threshold method.

To solve the above problem, we use the "token-controlled" approach. In the re-encryption key generation algorithm, we select a random token $\theta$ as the secret for the polynomial. And also we let $\theta$ involved in $I D_{j}$. When performing the re-encryption, the original ciphertext is "semi-decrypted" by the proxy using the re-encryption key. At last, the re-encrypted ciphertext is only relevant to the token $\theta$, which is encrypted under $I D_{j}$. Using $s k_{I D_{j}}$, he can recover $\theta$ and furthermore recover the message. 


\subsection{Modified Waters IBE scheme}

We first review the construction of modified Waters IBE scheme, which is named "Type-III mWIBE" in [3]. The details of the construction is shown as follows [3]:

1. $\operatorname{Setup}(\lambda)$ : Let $\lambda$ be the security parameter,and $\left(p, g, G, G_{t}, e\right)$ be the bilinear map parameters. Randomly choose $\alpha \in Z_{p}^{*}, g_{2}, u_{1}^{\prime}, u_{2}^{\prime}, u_{1,1}, \cdots, u_{1, n}, u_{2,0}, u_{2,1}, \cdots, u_{2, n} \in G$. Let PRF : $G_{T} \times G \rightarrow\{0,1\}^{l}$ be a pseudorandom function, and $(\mathcal{G}, \mathcal{S}, \mathcal{V})$ be a strongly unforgeable one-time signature. Set $g_{1}=g^{\alpha}$, and define two functions

$$
F_{1}(v)=u_{1}^{\prime} \prod_{i \in \mathcal{V}} u_{1, i}, \quad F_{2}(\omega)=u_{2}^{\prime} u_{2,0} \prod_{i \in \mathcal{W}} u_{2, i}
$$

where $v, \omega$ are two $n$-bit strings and $\mathcal{V}, \mathcal{W}$ are the set of all $i$ for which the $i$-th bit of $v$ and $\omega$ is one, respectively. The public key is $P P=\left(\lambda, g, g_{1}, g_{2}, F_{1}(\cdot), F_{2}(\cdot), P R F,(\mathcal{G}, \mathcal{S}, \mathcal{V})\right)$ and the mast secret key is $m s k=g_{2}^{\alpha}$.

2. $\operatorname{KeyGen}(m s k, I D)$ : Let $I D$ be an $n$-bit string, then the secret key for $I D$ is computed as

$$
s k_{I D}=\left(s k_{I D 1}, s k_{I D 2}\right)=\left(g_{2}^{\alpha} F_{1}(I D)^{r}, g^{r}\right),
$$

where $r$ is randomly chosen in $Z_{p}^{*}$.

3. $\operatorname{Enc}(I D, m)$ : Perform $\mathcal{G}(\lambda)$ to get a signature pair $(s s k, s v k)$. For a message $m \in\{0,1\}^{l_{1}}$, generate the ciphertext

$$
\begin{aligned}
C_{1} & =\left[\operatorname{PRF}\left(\sigma, C_{3}\right)\right]^{l-l_{1}} \|\left(\left[\operatorname{PRF}\left(\sigma, C_{3}\right)\right]_{l_{1}} \oplus m\right), \\
C_{2} & =\sigma \cdot e\left(g_{1}, g_{2}\right)^{t}, \\
C_{3} & =g^{t} \\
C_{4} & =F_{1}(I D)^{t} \\
C_{5} & =F_{2}(s v k)^{t} \\
S & =\mathcal{S}\left(s s k,\left(C_{1}, C_{3}, C_{4}, C_{5}\right)\right),
\end{aligned}
$$

where $t \stackrel{R}{\leftarrow} Z_{p}^{*}$, and $\sigma \stackrel{R}{\leftarrow} G_{T}$. Return the ciphertext $C=\left(s v k, C_{1}, C_{2}, C_{3}, C_{4}, C_{5}, S\right)$.

4. $\operatorname{Dec}\left(s k_{I D}, C\right)$ : Verify $\mathcal{V}\left(s v k, S,\left(C_{1}, C_{3}, C_{4}, C_{5}\right)\right) \stackrel{?}{=} 1, e\left(g, C_{4}\right) \stackrel{?}{=} e\left(C_{3}, F_{1}(I D)\right)$ and $e\left(g, C_{5}\right) \stackrel{?}{=} e\left(C_{3}, F_{2}(s v k)\right)$. If the equations do not hold, output $\perp$. Otherwise compute $\sigma=\frac{C_{2} \cdot e\left(s k_{I D_{2}}, C_{4}\right)}{e\left(s k_{I D_{1}}, C_{3}\right)}$. Return $m=\left[\operatorname{PRF}\left(\sigma, C_{3}\right)\right]_{l_{1}} \oplus\left[C_{1}\right]_{l_{1}}$, if $\left[C_{1}\right]^{l-l_{1}}=\left[\operatorname{PRF}\left(\sigma, C_{3}\right)\right]^{l_{1}}$. Otherwise output $\perp$.

We refer to the above modified Waters IBE as mWIBE. As stated in [3], the above mWIBE is secure against CCA. Thus we have the following theorem.

Theorem 1. The mWIBE scheme is CCA secure under the DBDH assumption if PRF is a pseudorandom function and $(\mathcal{G}, \mathcal{S}, \mathcal{V})$ is a strongly unforgeable one-time signature. 


\subsection{Our construction}

We first define the Lagrange coefficient $\Delta_{\omega, S}(x)$ for $\omega \in Z_{p}$ and a set $S$, of elements in $Z_{p}$ :

$$
\Delta_{\omega, S}(x)=\prod_{i \in S, i \neq \omega} \frac{x-i}{\omega-i} .
$$

Our proposed IB-CPRE-FG consists of the following algorithms:

1. $\operatorname{Setup}(\lambda)$ : Let $\lambda$ be the security parameter,and $\left(p, g, G, G_{T}, e\right)$ be the bilinear map parameters. Randomly choose $\alpha, \varepsilon \in Z_{p}^{*}, g_{2}, Z, u_{1}^{\prime}, u_{2}^{\prime}, u_{3}^{\prime}, u_{3,0}, u_{1,1}, \cdots, u_{1, n}, u_{2,1}, \cdots, u_{2, n}$, $u_{3,1}, \cdots, u_{3, n} \in G$. Let $P R F: G_{T} \times G \rightarrow\{0,1\}^{l}$ be a pseudorandom function, $H_{1}:\{0,1\}^{k_{1}} \rightarrow Z_{p}^{*}, H_{2}:\{0,1\}^{k_{2}} \rightarrow G$ be collision-resistent hash functions. Denote $l_{1}=k_{1}+k_{2}$. Let $(\mathcal{G}, \mathcal{S}, \mathcal{V})$ be a strongly unforgeable one-time signature. Set $g_{1}=g^{\alpha}$, and define three functions

$$
F_{1}(v)=u_{1}^{\prime} \prod_{i \in \mathcal{V}} u_{1, i}, \quad F_{2}(\omega)=u_{2}^{\prime} \prod_{i \in \mathcal{W}} u_{2, i}, \quad F_{3}(x)=u_{3}^{\prime} u_{3,0} \prod_{i \in \mathcal{X}} u_{3, i}
$$

where $v, \omega, x$ are three $n$-bit strings and $\mathcal{V}, \mathcal{W}, \mathcal{X}$ are the set of all $i$ for which the $i$-th bit of $v, \omega$ and $x$ is one, respectively. The public key is

$$
P P=\left(\lambda, l, k_{1}, k_{2}, g, g_{1}, g_{2}, g^{\varepsilon}, Z, F_{1}(\cdot), F_{2}(\cdot), F_{3}(\cdot), P R F, H,(\mathcal{G}, \mathcal{S}, \mathcal{V})\right)
$$

and the master secret key is $m s k=g_{2}^{\alpha}$.

2. KeyGen $(m s k, I D)$ : Let $I D$ be an $n$-bit string, then the secret key for $I D$ is computed as

$$
s k_{I D}=\left(s k_{I D 1}, s k_{I D 2}\right)=\left(g_{2}^{\alpha} F_{1}(I D)^{r}, g^{r}\right),
$$

where $r$ is randomly chosen in $Z_{p}^{*}$.

3. $\operatorname{Enc}(I D, m, W)$ : To encrypt a message $m \in M$, under identity $I D$ and a keyword set $W$ at the second level, the sender proceeds as follows:

(a) Select a one-time signature key pair as $(s s k, s v k) \leftarrow \mathcal{G}(\lambda)$.

(b) Set $C_{0}=W$.

(c) Select a random value $t \stackrel{R}{\leftarrow} Z_{p}^{*}$ and compute

$$
\begin{gathered}
C_{1}=\left[\operatorname{PRF}\left(\sigma, C_{3}\right)\right]^{l-l_{1}} \|\left(\left[\operatorname{PRF}\left(\sigma, C_{3}\right)\right]_{l_{1}} \oplus m\right), \quad C_{2}=\sigma \cdot e\left(g_{1}, g_{2}\right)^{t}, \\
C_{3}=g^{t}, \quad C_{4}=F_{1}(I D)^{t}, \quad C_{5}=\left(C_{\omega}=F_{2}(\omega)^{t}\right)_{\omega \in W}, \\
C_{6}=F_{3}(s v k)^{t}, \quad C_{7}=\left(g^{\varepsilon}\right)^{t} .
\end{gathered}
$$

(d) Run the signing algorithm $\mathcal{S}\left(s s k,\left(C_{1}, C_{3}, C_{4}, C_{5}, C_{6}\right)\right)$ and denote the signature as $S$.

(e) Output the ciphertext $C T_{I D}^{(2)}=\left(s v k, C_{0}, C_{1}, C_{2}, C_{3}, C_{4}, C_{5}, C_{6}, C_{7}, S\right)$. 
4. RKeyGen $\left(s k_{I D_{i}}, I D_{i}, \mathcal{T}, I D_{j}\right)$ : On input two identities $I D_{i}, I D_{j}$, an access tree $\mathcal{T}$, and user $I D_{i}^{\prime} s$ private key $s k_{I D_{i}}$. The ReKeyGen algorithms outputs a re-encryption key $r k_{\mathcal{T} \mid I D_{i} \rightarrow I D_{j}}$ which enables the proxy to re-encrypted a ciphertext under a condition set $W$, if and only if $\mathcal{T}(W)=1$. The algorithms proceeds as follows. First chooses random values $\theta_{1} \in\{0,1\}^{k_{1}}, \theta_{2} \in\{0,1\}^{k_{2}}$, also chooses a polynomial $q_{x}$ for each non-leaf node $x$ in the tree $\mathcal{T}$. These polynomials are chosen in the following way in a top down manner, starting from the root node $r$. It calls the procedure $R K G\left(\mathcal{T}, H_{1}\left(\theta_{1}\right)\right)$, where procedure $R K G\left(\mathcal{T}, H_{1}\left(\theta_{1}\right)\right)$ is defined as follows:

For each node $x$ in the tree, set the degree $d_{x}$ of the polynomial $q_{x}$ to be one less than the threshold value $k_{x}$ of the note, that is, $d_{x}=k_{x}-1$. Now for the root $r$, set $q_{r}(0)=H_{1}\left(\theta_{1}\right)$ and $d_{r}$ other points of the polynomial $q_{r}$ randomly to define it completely. For any other node $x$, set $q_{x}(0)=q_{p(x)}($ index $(x))$ and choose $d_{x}$ other points randomly to completely define $q_{x}$, once the polynomials have been decided, for each leaf node $x$, set $\omega=$ keyword $(x)$. Thus the re-encryption key given to the proxy are calculated as follows:

(a) Select a random value $s_{x} \stackrel{R}{\longleftarrow} Z_{p}^{*}$, computes

$$
a_{x}=Z^{q_{x}(0)} F_{2}(\omega)^{s_{x}} \quad b_{x}=g^{s_{x}}
$$

Let $a=\left\{a_{x}: x \in L N_{\mathcal{T}}\right\}$ and $b=\left\{b_{x}: x \in L N_{\mathcal{T}}\right\}$.

(b) Select a one-time signature key pair as $\left(s s k^{\prime}, s v k^{\prime}\right) \leftarrow \mathcal{G}(\lambda)$.

(c) Select random value $\rho, t^{\prime} \stackrel{R}{\leftarrow} Z_{p}^{*}$ and $\sigma^{\prime} \stackrel{R}{\leftarrow} G_{T}$, compute

$$
\begin{gathered}
r k_{0}=s k_{I D_{i, 1}} \cdot\left(g^{\varepsilon}\right)^{\rho}, \quad r k_{1}=g^{\rho}, \quad r k_{2}=s k_{I D_{i, 2}} \cdot H_{2}\left(\theta_{2}\right), \\
r k_{3}=\left[P R F\left(\sigma^{\prime}, r k_{5}\right)\right]^{l-l_{1}} \|\left(\left[P R F\left(\sigma^{\prime}, r k_{5}\right)\right]_{l_{1}} \oplus\left(\theta_{1} \| \theta_{2}\right)\right), \quad r k_{4}=\sigma^{\prime} \cdot e\left(g_{1}, g_{2}\right)^{t^{\prime}}, \\
r k_{5}=g^{t^{\prime}}, \quad r k_{6}=F_{1}\left(I D_{j}\right)^{t^{\prime}}, \quad r k_{7}=F_{3}\left(s v k^{\prime}\right)^{t^{\prime}} .
\end{gathered}
$$

(d) Run the signing algorithm $\mathcal{S}\left(s s k^{\prime},\left(r k_{3}, r k_{5}, r k_{6}, r k_{7}\right)\right)$ and denote the signature $S^{\prime}$.

(e) Return $r k_{\mathcal{T} \mid I D_{i} \rightarrow I D_{j}}=\left(a, b, \mathcal{T}, s v k^{\prime}, r k_{0}, r k_{1}, r k_{2}, r k_{3}, r k_{4}, r k_{5}, r k_{6}, r k_{7}, S^{\prime}\right)$.

5. $\operatorname{ReEnc}\left(C_{I D_{i}}^{(2)}, r k_{\mathcal{T} \mid I D_{i} \rightarrow I D_{j}}\right):$ On input a re-encryption key $r k_{\mathcal{T} \mid I D_{i} \rightarrow I D_{j}}=\left(a, b, \mathcal{T}, s v k^{\prime}\right.$, $\left.r k_{0}, r k_{1}, r k_{2}, r k_{3}, r k_{4}, r k_{5}, r k_{6}, r k_{7}, S^{\prime}\right)$ and a second level ciphertext $C_{I D_{i}}^{(2)}=\left(s v k, C_{0}, C_{1}\right.$, $\left.C_{2}, C_{3}, C_{4}, C_{5}, C_{6}, S\right)$ under the key word set $W$. Recover $W=C_{0}$, for all $\omega \in W$ it check whether the following equalities hold:

$$
\begin{array}{r}
\mathcal{V}\left(s v k, S,\left(C_{1}, C_{3}, C_{4}, C_{5}, C_{6}\right)\right) \stackrel{?}{=} 1, \\
e\left(g, C_{4}\right) \stackrel{?}{=} e\left(C_{3}, F_{1}\left(I D_{i}\right)\right), \\
e\left(g, C_{6}\right) \stackrel{?}{=} e\left(C_{3}, F_{3}(s v k)\right), \\
e\left(g, C_{\omega}\right)_{\omega \in W} \stackrel{?}{=} e\left(C_{3}, F_{2}(\omega)\right)_{\omega \in W}, \\
e\left(g, C_{7}\right) \stackrel{?}{=} e\left(C_{3}, g^{\varepsilon}\right) .
\end{array}
$$

If not, output $\perp$. Otherwise, we define a recursive algorithms $\operatorname{Node} \operatorname{Re} \operatorname{Enc}\left(C_{I D_{i}}^{(2)}\right.$, $\left.r k_{\mathcal{T} \mid I D_{i} \rightarrow I D_{j}}, x\right)$ that takes as input the ciphertext $C_{I D_{i}}^{(2)}$, the re-encryption key $r k_{\mathcal{T} \mid I D_{i} \rightarrow I D_{j}}$, and a note $x$ in the tree. 
(a) For leaf node $x$, let $\omega=\operatorname{keyword}(x)$, if $\omega \in W$, then

$$
\begin{aligned}
& \text { NodeReEnc }\left(C_{I D_{i}}^{(2)}, r k_{\mathcal{T} \mid I D_{i} \rightarrow I D_{j}}, x\right) \\
& =\frac{e\left(C_{3}, a_{x}\right)}{e\left(b_{x}, C_{\omega}\right)} \\
& =\frac{e\left(g^{t}, Z^{q_{x}(0)} F_{2}(\omega)^{s_{x}}\right)}{e\left(g^{s_{x}}, F_{2}(\omega)^{t}\right)} \\
& =e(g, Z)^{t q_{x}(0)}
\end{aligned}
$$

Otherwise, outputs NodeReEnc $\left(C_{I D_{i}}^{(2)}, r k_{\mathcal{T} \mid I D_{i} \rightarrow I D_{j}}, x\right)=\perp$.

(b) If $x$ is a non-leaf node, consider the recursive case, for all nodes $z$ that are children of $x$, it calls $N o d e \operatorname{Re} \operatorname{Enc}\left(C_{I D_{i}}^{(2)}, r k_{\mathcal{T} \mid I D_{i} \rightarrow I D_{j}}, z\right)$ and stores the output as $F_{z}$. Let $S_{x}$ be an arbitrary $k_{x}$-sized set of child notes $z$, such that $F_{z} \neq \perp$. If no such set exists, then the node was not satisfied and the function $\operatorname{NodeRe} \operatorname{Enc}\left(C_{I D_{i}}^{(2)}, r k_{\mathcal{T} \mid I D_{i} \rightarrow I D_{j}}, x\right)$ returns $\perp$. Otherwise, let $S_{x}^{\prime}=\left\{\operatorname{index}(z): z \in S_{)}\right\}$, and computes

$$
\begin{aligned}
F_{x} & =\prod_{z \in S_{x}, i=i n d e x(z)}\left(F_{z}\right)^{\Delta_{i, S_{x}^{\prime}}(0)} \\
& =\prod_{z \in S_{x}, i=i n d e x(z)}\left(e(g, Z)^{t q_{z}(0)}\right)^{\Delta_{i, S_{x}^{\prime}}(0)} \\
& =\prod_{z \in S_{x}, i=i n d e x(z)}\left(e(g, Z)^{t q_{p(z)}(i n d e x(z))}\right)^{\Delta_{i, S_{x}^{\prime}}(0)} \\
& =\prod_{z \in S_{x}, i=i n d e x(z)}\left(e(g, Z)^{t q_{x}(i)}\right)^{\Delta_{i, S_{x}^{\prime}}(0)} \\
& =e(g, Z)^{t q_{x}(0)}
\end{aligned}
$$

and return the result. Then we can compute

$$
\begin{aligned}
F_{r} & =N o d e R e E n c\left(C_{I D_{i}}^{(2)}, r k_{\mathcal{T} \mid I D_{i} \rightarrow I D_{j}}, r\right) \\
& =e(g, Z)^{t q_{r}(0)} \\
& =e(g, Z)^{t H_{1}\left(\theta_{1}\right)}
\end{aligned}
$$

(c) Compute $C_{2}^{\prime}=\frac{C_{2} \cdot F_{r} \cdot e\left(r k_{2}, C_{4}\right)}{e\left(r k_{0}, C_{3}\right) / e\left(r k_{1}, C_{7}\right)}$.

Finally, output the re-encrypted ciphertext

$$
C T_{I D_{j}}^{(1)}=\left(s v k, C_{1}, C_{2}^{\prime}, C_{3}, C_{4}, C_{5}, C_{6}, S, s v k^{\prime}, r k_{3}, r k_{4}, r k_{5}, r k_{6}, r k_{7}, S^{\prime}\right) .
$$

6. $\operatorname{Dec} 2\left(I D_{i}, s k_{I D_{i}}, C T_{I D_{i}}^{(2)}\right)$ :

(a) First check the validity of the ciphertexts as in equations (1)-(5). If the verification fails, output $\perp$ and abort.

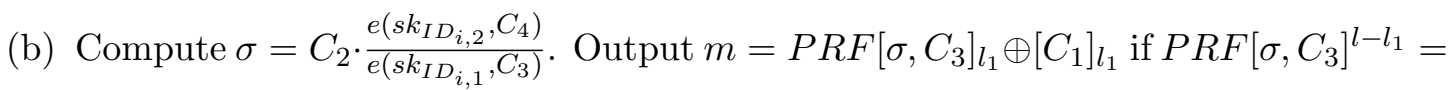
$\left[C_{1}\right]^{l-l_{1}}$, else output $\perp$ and abort. 
7. $\operatorname{Dec} 1\left(I D_{j}, s k_{I D_{j}}, C T_{I D_{j}}^{(1)}\right)$ :

(a) Verify

$$
\begin{array}{r}
\mathcal{V}\left(s v k^{\prime}, S^{\prime},\left(r k_{3}, r k_{5}, r k_{6}, r k_{7}\right)\right) \stackrel{?}{=} 1, \\
e\left(g, r k_{6}\right) \stackrel{?}{=} e\left(r k_{5}, F_{1}\left(I D_{j}\right)\right), \\
e\left(g, r k_{7}\right) \stackrel{?}{=} e\left(r k_{5}, F_{3}\left(s v k^{\prime}\right)\right) .
\end{array}
$$

If the equations (6)-(8) do not hold, output $\perp$ and abort. Otherwise, proceed.

(b) Compute $\sigma^{\prime}=r k_{4} \cdot \frac{e\left(s k_{\left.I D_{j, 2}, r k_{6}\right)}\right.}{e\left(s k_{I D_{j, 1}}, r k_{5}\right)}$. Output $\theta_{1} \| \theta_{2}=P R F\left[\sigma^{\prime}, r k_{5}\right]_{l_{1}} \oplus\left[r k_{3}\right]_{l_{1}}$ if $\operatorname{PRF}\left[\sigma^{\prime}, r k_{5}\right]^{l-l_{1}}=\left[r k_{3}\right]^{l-l_{1}}$, else output $\perp$ and abort.

(c) Verify equations (1)-(4). If (1)-(4) do not hold output $\perp$ and abort. Otherwise, proceed.

(d) Compute $\sigma=C_{2}^{\prime} /\left(e\left(C_{3}, Z\right)^{H_{1}\left(\theta_{1}\right)} \cdot e\left(C_{4}, H_{2}\left(\theta_{2}\right)\right)\right)$. Output $m=P R F\left[\sigma, C_{3}\right]_{l_{1}} \oplus$ $\left[C_{1}\right]_{l_{1}}$ if $\operatorname{PRF}\left[\sigma, C_{3}\right]^{l-l_{1}}=\left[C_{1}\right]^{l-l_{1}}$, else output $\perp$ and abort.

Correctness. We now explain the correctness of our scheme:

1. For an original ciphertext $C T_{I D}^{(2)}=\left(s v k, C_{0}, C_{1}, C_{2}, C_{3}, C_{4}, C_{5}, C_{6}, S\right)$, we have

$$
\begin{aligned}
& C_{2} \cdot \frac{e\left(s k_{I D_{i, 2}}, C_{4}\right)}{e\left(s k_{I D_{i, 1}}, C_{3}\right)}=\sigma \cdot e\left(g_{1}, g_{2}\right)^{t} \cdot \frac{e\left(g^{r}, F_{1}\left(I D_{i}\right)^{t}\right)}{e\left(g_{2}^{\alpha} \cdot F_{1}\left(I D_{i}\right)^{r}, g^{t}\right)} \\
& =\sigma \cdot e\left(g_{1}, g_{2}\right)^{t} \cdot \frac{1}{e\left(g_{2}^{\alpha}, g^{t}\right)} \\
& =\sigma .
\end{aligned}
$$

2. For a re-encrypted ciphertext $C T_{I D_{j}}^{(1)}=\left(s v k, C_{1}, C_{2}^{\prime}, C_{3}, C_{4}, C_{6}, S, s v k^{\prime}, r k_{3}, r k_{4}, r k_{5}, r k_{6}\right.$, $\left.r k_{7}, S^{\prime}\right)$, we have

$$
\begin{aligned}
C_{2}^{\prime} & =\frac{C_{2} \cdot F_{r} \cdot e\left(r k_{2}, C_{4}\right)}{e\left(r k_{0}, C_{3}\right) / e\left(r k_{1}, C_{4}\right)} \\
& =\frac{\sigma \cdot e\left(g_{1}, g_{2}\right)^{t} \cdot e(g, Z)^{t H_{1}\left(\theta_{1}\right)} \cdot e\left(g^{r} \cdot H_{2}\left(\theta_{2}\right), F_{1}\left(I D_{i}\right)^{t}\right)}{e\left(g_{2}^{\alpha} F_{1}\left(I D_{i}\right)^{r} \cdot\left(g^{\varepsilon}\right)^{\rho}, g^{t}\right) / e\left(g^{\rho},\left(g^{\varepsilon}\right)^{t}\right)} \\
& =\sigma \cdot e\left(C_{4}, H_{2}\left(\theta_{2}\right)\right) \cdot e(g, Z)^{t H_{1}\left(\theta_{1}\right)}
\end{aligned}
$$

Then we have $\sigma=C_{2}^{\prime} /\left(e\left(C_{3}, Z\right)^{H_{1}\left(\theta_{1}\right)} \cdot e\left(C_{4}, H_{2}\left(\theta_{2}\right)\right)\right)$, where $\theta_{1}, \theta_{2}$ can be correctly computed using the same way as above,

\subsection{Security of our key-private IBPRE scheme}

Theorem 2. Our IB-CPRE-FG scheme is IND-level2-CCA secure assuming the DBDH assumption holds, $P R F$ is a pseudorandom function, $H_{1}, H_{2}$ are collision-resistent hash functions and $(\mathcal{G}, \mathcal{S}, \mathcal{V})$ is a strongly unforgeable one-time signature. 
The proof of Theorem 2 is provided in Appendix A.

Theorem 3. Our IB-CPRE-FG scheme is IND-level1-CCA secure assuming the DBDH assumption holds, $P R F$ is a pseudorandom function, $H_{1}, H_{2}$ are collision-resistent hash functions and $(\mathcal{G}, \mathcal{S}, \mathcal{V})$ is a strongly unforgeable one-time signature.

The proof of Theorem 3 is provided in Appendix B.

\section{Comparison and discussion}

\subsection{Comparison}

In this section, we compare our scheme with previous IB-CPRE schemes in terms of private/reencryption key size, levekl2/level1 ciphertext size, re-encryption cost, properties and security model in Table 1. Let $g, g_{t}$ and $z$ be the size of an element in $G, G_{T}$ and $Z_{p}$, respectively. Let $m$ be the size of a message $M$ with $m$ bit in $\{0,1\}^{*}, S$ be the size of a strongly unforgeable one-time signature, $t$ be the size of an access tree and $w$ be the size of attributes used in the encryption algorithm. Besides, $c_{e}, c_{p}$ and $v$ denote the computational cost of an exponentiation, a bilinear pairing and the verification of one-time signature.

\begin{tabular}{|c|c|c|c|c|}
\hline Schemes & $\begin{array}{l}\text { Priavte/Re-encry- } \\
\text { ption key size }\end{array}$ & $\begin{array}{l}\text { level } 2 / \text { level } 1 \\
\text { ciphertext size }\end{array}$ & $\begin{array}{l}\text { Re-encryp- } \\
\text { tion cost }\end{array}$ & $\begin{array}{l}\text { without RO? } \\
\text { /fine grain policy? }\end{array}$ \\
\hline IB-CPRE [2] & $g / 3 g+m$ & $\begin{array}{l}3 g+g_{t}+m \\
/ 3 g+g_{t}+m\end{array}$ & $2 c_{p}$ & $\times / \times$ \\
\hline IB-CPRE [3] & $2 g / 6 g+g_{t}+S$ & $\begin{array}{c}4 g+g_{t}+m+S \\
/ 7 g+2 g_{t}+m+2 S\end{array}$ & $9 c_{p}+v$ & $\sqrt{ } / \times$ \\
\hline Our IB-PRE-FG & $\begin{array}{c}2 g \\
/ 6 g+g+t+S+m+t\end{array}$ & $\begin{array}{l}(w+4) g+g_{t}+m+w+S \\
/(w+6) g+2 g_{t}+2 m+2 S\end{array}$ & $(9+2 w+t) c_{p}+v$ & $\sqrt{ } / \sqrt{ }$ \\
\hline
\end{tabular}

Table 1. Comparison with Shao et al.'s scheme [2] and Liang et al.'s scheme [3]

\subsection{Master secret security}

Master secret security means that even if a proxy colludes with a set of delegatees, it is still impossible for them to expose the delegator's private key [10]. As discussed in [12], the notion of CCA security of level 1 ciphertext implies the master secret security. In our proposed scheme, a collusion of proxy and delegatee can only extract the second part $s k_{I D_{i}, 2}$ of the private key of the delegator. Additionally, the first part $s k_{I D_{i}, 1}$ remains secret to the proxy and delegatee.

\subsection{Non-transitivity and non-transferability issue}

Non-transitivity means that the proxy alone cannot re-delegate the decryption rights. For example, from $r k_{I D_{i} \rightarrow I D_{j}}$ and $r k_{I D_{j} \rightarrow I D_{k}}$, he cannot produce $r k_{I D_{i} \rightarrow I D_{k}}$. Non-transferability means the proxy and a set of colluding delegatees cannot re-delegate decryption rights. For example, from $r k_{I D_{i} \rightarrow I D_{j}}, s k_{I D_{j}}$ and $I D_{j}$, they cannot produce $r k_{I D_{i} \rightarrow I D_{j}}$. Note that, in our scheme, user $I D_{j}$ can produce the re-encryption key $s k_{I D_{j} \rightarrow I D_{k}}$, so non-transitivity is implied by non-transferability. Our scheme is transferable, as the collusion of the proxy with $r k_{I D_{i} \rightarrow j}$ 
and a delegatee with $s k_{I D_{j}}$ can produce a new re-encryption key $r k_{I D_{i} \rightarrow I D_{k}}$. As discussed in [10], transferability is "mild-harmful", as $I D_{j}$ can always decrypt and forward the plaintext to $I D_{j}$. However, this requires that $I D_{j}$ should be online. Achieving an identity-based conditional proxy re-encryption with fine-grain policy scheme that is non-transferable seems to be an interesting future work.

\section{Conclusions}

In this work, we give an affirmative answer to an existing open problem by presenting an a new IB-CPRE-FG scheme. We formulated the security model of IB-CPRE-FG and proved its IND-CCA security. Many interesting questions still remain to be solved though. In our scheme, the access policy is decscribed by an access structure. First, it is interesting to construct an IB-CPRE scheme supporting AND or OR gates directly. Second, as many proxy re-encryption schemes [36, 37] have been proposed to capture the key-private property, hence, it is also an interesting open problem to construct a key-private IB-CPRE scheme.

\section{Acknowledgement}

This work is supported by the National Natural Science Foundation of China (No. 61272083, 61300236, 61672270, 61602216), the National Natural Science Foundation of Jiangsu (No. BK20130809), the National Science Foundation for Post-doctoral Scientists of China (No. 2013M530254), the National Science Foundation for Post-doctoral Scientists of Jiangsu (No. 1302137C), the China Postdoctoral Science special Foundation (No. 2014T70518), the Research Foundation for Humanities and Social Sciences of Ministry of Education, China (No. 14YJAZH023,15YJCZH129), and the Qing Lan Project for Young Researchers of Jiangsu Province of China(No. KYQ14004).

\section{References}

[1] Green, M., Ateniese, G. (2007) Identity-based proxy re-encryption. Proceedings of ACNS 2007, Zhuhai, China, 5-8 June, pp.288-306. Springer-Verlag, Berlin.

[2] Shao, J., Wei, G., Ling, Y., Xie, M. (2011) Identity-based Conditional Proxy Reencryption. Proceedings of IEEE ICC 2011, Kyoto, Japan, 5-9 June, pp.1-5. IEEE, USA.

[3] Liang, K., Liu, Z., Tan, X., Wong D.S., Tang, C., (2013) A CCA-Secure Identity-Based Conditional Proxy Re-Encryption without Random Oracles. Proceedings of ICISC 2012, Seoul, Korea, 28-30 November, pp.231-246. Springer-Verlag, Berlin.

[4] Bethencourt, j., Sahai, A., Waters, B. (2007) Ciphertext-policy attribute-based encrtption. Proceedings of IEEE Symposium on Security and Privacy 2007, Berkeley, California, 20-23 May, pp.321-334. IEEE Computer Society, Washington, DC, USA.

[5] Liang, X., Cao, Z., Lin, H., Shao, J. (2009) Attribute-Based Proxy Re-Encryption with Delegating Capabilities. Proceedings of ASIACCS 2009, Sydney, Australia, 10-12 March, pp.276-286. ACM, NewYork. 
[6] Luo, S., Hu, J., Chen, Z. (2010) Ciphertext policy attribute-based proxy re-encryption. Proceedings of ICICS 2010, Barcelona, Spain, 15-17 December, pp.401-415. SpringerVerlag, Berlin.

[7] Mizuno, T., Doi, H. (2011) Hybrid proxy re-encryption scheme for attribute-based encryption. Proceedings of ICISC 2011, Seoul, Korea, 30 November - 2 December, pp.288302. Springer-Verlag, Berlin.

[8] Fang, L., Susilo, W., Ge, C., Wang, J. (2011) Interactive conditional proxy re-encryption with fine grain policy. Journal of Systerms and Software, 84, 2293-2302.

[9] Blaze, M., Bleumer, G., Strauss, M. (1998) Divertible protocols and atomic proxy cryptography. Proceedings of EUROCRYPT 1998, Finland, 31 May 31 - 4 June, pp.127-144. Springer-Verlag, Berlin.

[10] Ateniese, G., Fu, K., Green, M., Hohenberger, S. (2005) Improved proxy re-encryption schemes with applications to secure distributed storage. Proceedings of the 12th Annual Network and Distributed System Security Symposium 2005, San Diego, California, USA, 3-4 February, pp.29-44. ACM, NewYork.

[11] Canetti, R., Hohenberger, S. (2007) Chosen-ciphertext secure proxy re-encryption. Proceedings of the 14th ACM conference on Computer and Comuniation, Security 2007, 29-31 October-1 November, pp.185-194. ACM, NewYork.

[12] Libert, B., Vergnaud, D. (2008) Unidirectional chosen-ciphertext secure proxy reencryption. Proceedings of PKC 2008, Barcelona, Spain, 9-12 March, pp.360-379. Springer-Verlag, Berlin.

[13] Weng, J., Chen, M., Yang, Y., DENG, R., Chen, K., Bao, F. (2010) CCA-secure unidirectional proxy re-encryption in the adaptive corruption model without random oracles. Journal of Sci China Inf Sci, 53, 593-606.

[14] Shao, J., Lu, R., Lin, X., Liang, K. (2015) Secure bidirectional proxy re-encryption for cryptographic cloud storage. Pervasive and Mobile Computing, 28, 113-121.

[15] Lu, R., Lin, X., Shao, J., Liang, K. (2014) RCCA-Secure Multi-use Bidirectional Proxy Re-encryption with Master Secret Security. Proceedings of Provable Security 2014, HongKong, China, 9-10 October, pp.194-205. LNCS.

[16] Weng, J., Deng, R.H., Chu, C. (2009) Conditional proxy re-encryption secure against chosen-ciphertext attack. Proceedings of the 4th International Symposium on ACM Symposium on Information, Computer and Communications Security 2009, Sydeny, Australia, 10-12 March, pp.322-332. ACM, NewYork.

[17] Fang, L., Susilo, W., Ge, C., Wang, J. (2012) Chosen-ciphertext secure anonymous conditional proxy re-encryption with keyword search. Theoretical Computer Science, $462,39-58$.

[18] Liang, K. (2015) Efficient and Fully CCA Secure Conditional Proxy Re-Encryption from Hierarchical Identity-Based Encryption. Computer Journal, 58, 2778-2792. 
[19] Chu, C., Tzeng, W. (2007) Identity-based proxy re-encryption without random oracles. Proceedings of ISC 2007, Valparaiso, Chile, 9-12 October, pp.189-202. Springer-Verlag, Berlin.

[20] Waters, B. (2005) Efficient identity-based encryption without random oracles. Proceedings of EUROCRYPT 2005, Aarhus, Denmark, 22-26 May, pp.189-202. Springer-Verlag, Berlin.

[21] Liang, K., Chu, C.K., Tan, X., Wong, D.S., Tang, C., Zhou, J. (2014) Chosen-ciphertext secure multi-hop identity-based conditional proxy re-encryption with constant-size ciphertexts. Theoretical Computer Science, 539:87-105.

[22] Liang, K., Susilo, W., Liu, J.K. (2015) Privacy-Preserving Ciphertext Multi-Sharing Control for Big Data Storage. IEEE Transactions on Information Forensics and Security, 10(8):1578-1589.

[23] Liang, K., Fang, L., Wong, D.S., Susilo, W. (2014) A ciphertext-policy attribute-based proxy re-encryption scheme for data sharing in public clouds. Concurrency and Computation: Practice and Experience, 27, 2004-2027.

[24] Liang, K., Man, H.A., Liu, J.K., Susilo, W., Wong, D.S., Yang, G., Yu, Y., Yang, A. (2015) A secure and efficient Ciphertext-Policy Attribute-Based Proxy Re-Encryption for cloud data sharing. Future Generation Compupter Systems, 52, 95-108.

[25] Liang, K., Susilo, W. (2015) Searchable Attribute-Based Mechanism With Efficient Data Sharing for Secure Cloud Storage. IEEE Transactions on Information Forensics and Security, 10(9):1981-1992.

[26] Liang, K., Su, C., Chen, J., Liu, J.K. (2016) Efficient Multi-Function Data Sharing and Searching Mechanism for Cloud-Based Encrypted Data. Proceedings of ASIA CCS2016, Xi'an, China, 30 May-3 June, pp.83-94. ACM, USA.

[27] Ge, C., Susilo, W., Huang, Z., Wang, J., Fang, L., Ren,. (2016) A Key-Policy Attributebased Proxy Re-encryption without random oracles. Computer Journal, 59(7), pp.970982. Oxford University press, London.

[28] Liang, K., Au, M.H., Liu, J.K., Susilo, W., Wong, D.S., Yang, G., Phuong, T., Xie, Q. (2014) A DFA-Based Functional Proxy Re-Encryption Scheme for Secure Public Cloud Data Sharing. IEEE Transactions on Information Forensics and Security, 9(10):16671680 .

[29] Aono, Y., Boyen, X., Phong, X.T., Wang L., (2013) Key-Private Proxy Re-encryption unser LWE. Proceedings of INDOCRYPT 2013, Mumbai, India, 7-20 December, pp.1-18. Springer-Verlag, Berlin.

[30] Kirshanova, E., (2014) Proxy Re-encryption from lattices. Proceedings of PKC 2014, Buenos Aires, Argentina, 26-28 March, pp.77-94. Springer-Verlag, Berlin.

[31] Nunez, D., Agudo, I., Lopez, J., (2015) NTRUReEncrypt: An Efficient Proxy ReEncryption scheme Based on NTRU. Proceedings of ASIACCS 2015, Singapore, 14-17 April, pp.179-189. ACM, USA. 
[32] Boneh, D., Boyen, X. (2004) Efficient selective-ID based encryption without random oracles. Proceedings of EUROCRYPT 2004 Interlaken, Switzerland, 2-6 May, pp.223238. Springer-Verlag, Berlin.

[33] Boneh, D., Franklin, M. (2001) Identity-based encryption from the weil pairing. Proceedings of CRYPTO 2001, Santa Barbara, California, USA, 19-23 August, pp.231-229. Springer-Verlag, Berlin.

[34] Canetti, R., Halevi, S., Katz, J. (1999) Chosen-ciphertext security from identity-based encryption. Proceedings of PKC 1999, Kamakura, Japan, 21-23 May, pp.53-68. SpringerVerlag, Berlin.

[35] Goyal, V., Pandey, O., Sahai, A., Waters, B. (2006) Attribute-based encryption for finegrained access control of encrypted data. Proceedings of ACM CCS 2006, Alexandria, Virginia, USA, 30 October-3, November, pp.89-98. ACM, NewYork.

[36] Ateniese, G., Benson, K., Hohenberger, S. (2009) Key-private proxy re-encryption. Proceedings of RSA 2009, San Francisco, USA, 20-23 April, pp.279-294. Springer-Verlag, Berlin.

[37] Shao, J., Liu, P., Wei, G Y.,K.,Lin, Y. (2011) Anonymous proxy re-encryption. Journal of Security and Communication Networks, 10, 1002.

[38] Lai, J., Zhu, W., Deng, R. (2010) New constructions for identity-based unidirectional proxy re-encryption. Journal of Comput Sci Technol, 25, 793-806

[39] Tang, Q. (2008) Type-based proxy re-encryption and its construction. Proceedings of INDOCRYPT 2008, Kharagpur, India, 14-17 December, pp.130-144. Springer-Verlag, Berlin.

\section{A Proof of Theorem 2}

Proof. Suppose there is an adversary $\mathcal{A}$ who can break the IND-level2-CCA security of our IBCPRE-FG scheme with non-negligible probability $\epsilon$. We can construct a simulator $\mathcal{B}$ to break the mWIBE scheme with non-negligible probability $\epsilon^{\prime}$ using $\mathcal{A}$. Denote $\mathcal{B}_{1}$ as the challenge of the $\mathrm{mWIBE}$ in the CCA simulation. $\mathcal{B}$ first maintains the following tables which are initially empty.

- Key ${ }^{\text {List }}$ : records the tuples $\left(\beta, I D, S K_{I D}\right)$, which are the information of secret keys;

- ReKey ${ }^{\text {List }}$ : records the tuples $\left(\gamma, I D_{i}, \mathcal{T}, I D_{j}, r k_{\mathcal{T} \mid I D_{i} \rightarrow I D_{j}}, \theta_{1}, \theta_{2}, f l a g_{1}\right)$, which are the result of the queries to $\operatorname{RKExtract}\left(I D_{i}, \mathcal{T}, I D_{j}\right)$, where flag $=1$ denotes the reencryption key is a valid one, and $f l a g_{1}=0$ denotes the re-encryption key is a random value.

- ReEnc ${ }^{\text {List }}$ : records the tuples $\left(I D_{i}, I D_{j},\left(W, C_{\left(I D_{j}\right)}^{(2)}\right), C_{\left(I D_{j}\right)}^{(1)}, f l a g_{2}\right)$, which are the result of the queries to $\operatorname{ReEnc}\left(I D_{i}, I D_{j},\left(W, C_{I D_{i}}^{(2)}\right)\right)$, where $f l a g_{2}=1$ denotes the re-encrypted ciphertext is generated under a valid re-encryption key, and $f l a g_{2}=0$ denotes the reencrypted ciphertext is generated under a random re-encryption key. 
1. Setup: $\mathcal{B}$ first calls $\mathcal{B}_{1}$ to get the public parameter $P P=\left(\lambda, l, k_{1}, k_{2}, g, g_{1}, g_{2}, F_{1}(\cdot), F_{2}(\cdot)\right.$, $\left.F_{3}(\cdot), P R F,(\mathcal{G}, \mathcal{S}, \mathcal{V})\right), \mathcal{B}$ also chooses random values $\varepsilon \in Z_{p}^{*}, Z \in G$ and collisionresistent hash functions $H_{1}:\{0,1\}^{k_{1}} \longrightarrow Z_{p}^{*}, H_{2}:\{0,1\}^{k_{2}} \longrightarrow G$. Adds $g^{\varepsilon}, Z, H_{1}, H_{2}$ to $P P$ and forwards $P P$ to $\mathcal{A}$.

2. Query phase 1: $\mathcal{A}$ issues a series of queries to which $\mathcal{B}$ responds as follows:

(a) Extract $(I D): \mathcal{B}$ first searches Key ${ }^{\text {List }}$, if $\left(1, I D, s k_{I D}\right)$ exists in Key ${ }^{\text {List }}$, returns $s k_{I D}$ as the result. Otherwise, $\mathcal{B}$ generates a biased coin $\beta$ so that $\operatorname{Pr}[\beta=1]=\delta$ for some $\delta$ that will be determined later.

- If $\beta=0, \mathcal{B}$ outputs a random bit and aborts.

- If $\beta=1, \mathcal{B}$ forwards the query to $\mathcal{B}_{1}$ and obtains the secret key $s k_{I D}$. Returns $s k_{I D}$ to $\mathcal{A}$ and adds $\left(1, I D, s k_{I D}\right)$ to $K e y^{\text {List }}$.

(b) RKExtract $\left(I D_{i}, \mathcal{T}, I D_{j}\right): \mathcal{B}$ first searches whether there is a tuple $\left(*, I D_{i}, \mathcal{T}, I D_{j}\right.$, $\left.r k_{\mathcal{T} \mid I D_{i} \rightarrow I D_{j}}, \theta_{1}, \theta_{2}, *\right)$ in $\operatorname{ReKe} y^{\text {List }}$. If yes, $\mathcal{B}$ returns $r k_{\mathcal{T} \mid I D_{i} \rightarrow I D_{j}}$ as the result, where $*$ is the wildcard. Otherwise, $\mathcal{B}$ proceeds as follows:

- If $\left(1, I D_{i}, s k_{I D_{i}}\right)$ exists in Key ${ }^{\text {List }}, \mathcal{B}$ uses $s k_{I D_{i}}$ to generater the re-encryption key $r k_{\mathcal{T} \mid I D_{i} \rightarrow I D_{j}}$ via algorithm $R K e y G e n$ as in the real scheme. Returns the re-encryption key to $\mathcal{A}$ and adds $\left(*, I D_{i}, I D_{j}\right.$,

$\left.r k_{\mathcal{T} \mid I D_{i} \rightarrow I D_{j}}, \theta_{1}, \theta_{2}, 1\right)$ to ReKey ${ }^{\text {List }}$, where $\theta_{1} \in\{0,1\}^{k_{1}}$,

$\theta_{2} \in\{0,1\}^{k_{2}}$ are randomly chosen in the RKeyGen algorithm.

- Otherwise, $\mathcal{B}$ flips a biased coin $\beta$. If $\beta=1, \mathcal{B}$ queries the $\operatorname{Extract}\left(I D_{i}\right)$ oracle to get $s k_{I D_{i}}$, and then generates $r k_{\mathcal{T} \mid I D_{i} \rightarrow I D_{j}}$ via algorithm $R K$ eyGen as in the real scheme.

Returns the re-encryption key to $\mathcal{A}$ and adds $\left(1, I D_{i}, s k_{I D_{i}}\right)$ and $\left(*, I D_{i}, I D_{j}, r k_{\mathcal{T} \mid I D_{i} \rightarrow I D_{j}}, \theta_{1}, \theta_{2}, 1\right)$ to $K e y^{\text {List }}$ and ReKey ${ }^{\text {List }}$ respectively, where $\theta_{1} \in\{0,1\}^{k_{1}}, \theta_{2} \in\{0,1\}^{k_{2}}$ are randomly chosen in the $R K e y G e n$ algorithm. If $\beta=0, \mathcal{B}$ randomly chooses $\theta_{1} \in\{0,1\}^{k_{1}}, \theta_{2} \in$ $\{0,1\}^{k_{2}}, s_{x} \in Z_{p}^{*}$ and computer $a, b$ as in the real scheme. Then $\mathcal{B}$ sets $r k_{1}=\phi_{1}, r k_{2}=$ $\phi_{2}, r k_{3}=\phi_{3}$ for randomly chosen $\phi_{1}, \phi_{2}, \phi_{3} \in G$. Then $\mathcal{B}$ constructs $r k_{3}, r k_{4}, r k_{5}, r k_{6}, r k_{7}, s v k^{\prime}, S^{\prime}$ to encrypted a random $\left(\theta_{1} \| \theta_{2}\right) \in\{0,1\}^{l_{1}}$ as in the real scheme. $\mathcal{B}$ forwards the reencryption key to $\mathcal{A}$ and adds $\left(*, I D_{i}, \mathcal{T}, I D_{j}, r k_{\mathcal{T} \mid I D_{i} \rightarrow I D_{j}}, \theta_{1}, \theta_{2}, 0\right)$ to ReKey ${ }^{\text {List }}$.

(c) $\operatorname{Re} \operatorname{Enc}\left(I D_{i}, I D_{j},\left(W, C_{I D_{i}}^{(2)}\right)\right): \mathcal{B}$ first searches whether there is a tuple $\left(I D_{i}, I D_{j},(W\right.$, $\left.\left.C_{\left(I D_{j}\right)}^{(2)}\right), C_{\left(I D_{j}\right)}^{(1)}, *\right)$ in ReEnc ${ }^{\text {List }}$. If yes, $\mathcal{B}$ returns $C_{\left(I D_{j}\right)}^{(1)}$ as the result, where $*$ is the wildcard. Otherwise, $\mathcal{B}$ proceeds as follows:

- If $\left(*, I D_{i}, \mathcal{T}, I D_{j}, r k_{\mathcal{T} \mid I D_{i} \rightarrow I D_{j}}, \theta_{1}, \theta_{2}, *\right)$ exists in ReKey ${ }^{\text {List }}$, where $\mathcal{T}(W)=1$. $\mathcal{B}$ uses $r k_{\mathcal{T} \mid I D_{i} \rightarrow I D_{j}}$ to generater $C_{\left(I D_{j}\right)}^{(1)}$ via algorithm ReEnc as in the real scheme. Returns $C_{\left(I D_{j}\right)}^{(1)}$ to $\mathcal{A}$ and adds $\left(I D_{i}, I D_{j},\left(W, C_{\left(I D_{j}\right)}^{(2)}\right), C_{\left(I D_{j}\right)}^{(1)}, *\right)$ to ReEnc ${ }^{\text {List }}$.

- Otherwise, $\mathcal{B}$ first issues $R K \operatorname{Extract}\left(I D_{i}, \mathcal{T}, I D_{j}\right)$, where $\mathcal{T}(W)=1$ to obtain reencryption key. Next, generates $C_{\left(I D_{j}\right)}^{(1)}$ and adds $\left(I D_{i}, I D_{j},\left(W, C_{\left(I D_{j}\right)}^{(2)}\right), C_{\left(I D_{j}\right)}^{(1)}, *\right)$ to the ReEnc ${ }^{\text {List }}$.

(d) $\operatorname{Dec} 2\left(I D_{i},\left(W, C_{I D_{i}}^{(2)}\right)\right): \mathcal{B}$ first verifies equations (1)-(5). If the equations do not hold, output $\perp$ and abort. Otherwise, $\mathcal{B}$ proceeds:

- If $\left(1, I D_{i}, s k_{I D_{i}}\right)$ exists in $K e y^{\text {List }}$, using $s k_{I D_{i}}$ to recover $m$. 
- Otherwise, $\mathcal{B}$ forwards the query to the decryption oracle of mWIBE to obtain $m$.

(e) $\operatorname{Dec} 1\left(I D_{j}, C_{I D_{j}}^{(1)}\right): \mathcal{B}$ first verifies equations (1)-(4) and (6)-(8). If the equations do not hold, output $\perp$ and abort. Otherwise, $\mathcal{B}$ proceeds:

- If $\left(I D_{i}, I D_{j},\left(W, C_{\left(I D_{j}\right)}^{(2)}\right), C_{\left(I D_{j}\right)}^{(1)}, 1\right)$ exists in ReEnc ${ }^{L i s t}$, return $C_{\left(I D_{j}\right)}^{(2)}$ as the result.

- Else, $\mathcal{B}$ searches wether $\left(i, I D_{j}, s k_{I D_{j}}\right)$ exists in $K e y^{\text {List }}$, if yes, $\mathcal{B}$ recovers $m$ using $s k_{I D_{j}}$. Otherwise, $\mathcal{B}$ issues $\left(s v k^{\prime}, r k_{3}, r k_{4}, r k_{5}, r k_{6}, r k_{7}, S^{\prime}\right)$ to the decryption oracle of mWIBE and get $\theta_{1}, \theta_{2}$. Then using $\theta_{1}, \theta_{2}, \mathcal{B}$ can recover $m$ as in the real scheme.

3. Challenge: Once $\mathcal{A}$ decides that Phase 1 is over, it outputs two equal length message $\left(m_{0}, m_{1}\right)$, a target identity $I D^{*}$ and a target condition set $W^{*}$. $\mathcal{B}$ first checks wether the following two conditions hold:

- $\left(1, I D^{*}, s k_{I D^{*}}\right)$ does not exit in Key ${ }^{\text {List }}$;

- $\left(*, I D^{*}, \mathcal{T}, I D_{j}, r k_{\mathcal{T} \mid I D^{*} \rightarrow I D_{j}}, \theta_{1}, \theta_{2}, *\right)$, where $\mathcal{T}\left(W^{*}\right)=1$ and $\left(1, I D_{j}, s k_{I D_{j}}\right)$ do not exit in ReKey $y^{\text {List }}$ and Key ${ }^{\text {List }}$.

If the above two conditions do not hold, $\mathcal{B}$ outputs a random bit and aborts. Else, $\mathcal{B}$ forwards $\left(m_{0}, m_{1}, I D^{*}, W^{*}\right)$ to the challenge oracle of $\mathrm{mWIBE}$, and obtains the challenge ciphertext $C_{I D^{*}}^{(2) *}$.

4. Query phase 2: A continues making queries as in the query phase 1 with the restrictions described in the IND-level2-CCA game.

5. Guess: When A outputs a guess $b^{\prime} \in\{0,1\}, \mathcal{B}$ outputs $b^{\prime}$.

Probability analysis. If $\mathcal{B}$ does not abort, $\mathcal{A}$ 's view is identical to the real scheme. We define Abort be the event of $\mathcal{B}^{\prime} s$ aborting during the simulation of Extract query. Let $q_{e}$ denote the total number of Extract queries, we have $\operatorname{Pr}[\neg$ Abort $] \geq \delta^{q_{e}}(1-\delta)$, which is maximized at

$\delta_{\text {opt }}=\frac{q_{e}}{\left(1+q_{e}\right)}$. Using $\delta_{\text {opt }}$, the probability $\operatorname{Pr}[\neg A b o r t]$ is at least $\frac{1}{\dot{e}\left(1+q_{e}\right)}$, where $\dot{e}$ is the base of the nature logarithm. Therefor, we have

$$
\varepsilon^{\prime} \geq \frac{\varepsilon}{\dot{e}\left(1+q_{e}\right)}-A d v_{H_{1}, \mathcal{A}}^{T C R}-A d v_{H_{2}, \mathcal{A}}^{T C R}-A d v_{F, \mathcal{F}}^{P R F}
$$

This completes the proof of Theorem 2 .

\section{B Proof of Theorem 3}

Proof. Suppose there is an adversary $\mathcal{A}$ who can break the IND-level1-CCA security of our IB-CPRE-FG scheme with non-negligible probability $\varepsilon$. We can construct a simulator $\mathcal{B}$ to break the mWIBE scheme with non-negligible probability $\varepsilon^{\prime}$ using $\mathcal{A}$. Denote $\mathcal{B}_{1}$ as the challenge of the mWIBE in the CCA simulation.

1. Setup: $\mathcal{B}$ first calls $\mathcal{B}_{1}$ to get the public parameter $P P=\left(\lambda, l, k_{1}, k_{2}, g, g_{1}, g_{2}, F_{1}(\cdot), F_{2}(\cdot)\right.$, $\left.F_{3}(\cdot), P R F,(\mathcal{G}, \mathcal{S}, \mathcal{V})\right), \mathcal{B}$ also chooses random values $\varepsilon \in Z_{p}^{*}, Z \in G$ and collisionresistent hash functions $H_{1}:\{0,1\}^{k_{1}} \longrightarrow Z_{p}^{*}, H_{2}:\{0,1\}^{k_{2}} \longrightarrow G$. Adds $g^{\varepsilon}, Z, H_{1}, H_{2}$ to $P P$ and forwards $P P$ to $\mathcal{A}$. 
2. Query phase 1: $\mathcal{A}$ issues a series of queries to which $\mathcal{B}$ responds as follows:

(a) Extract $(I D): \mathcal{B}$ first searches Key ${ }^{\text {List }}$, if $\left(1, I D, s k_{I D}\right)$ exists in Key ${ }^{\text {List }}$, returns $s k_{I D}$ as the result. Otherwise, $\mathcal{B}$ generates a biased coin $\beta$ so that $\operatorname{Pr}[\beta=1]=\delta$ for some $\delta$ that will be determined later.

- If $\beta=0, \mathcal{B}$ outputs a random bit and aborts.

- If $\beta=1, \mathcal{B}$ forwards the query to $\mathcal{B}_{1}$ and obtains the secret key $s k_{I D}$. Returns $s k_{I D}$ to $\mathcal{A}$ and adds $\left(1, I D, s k_{I D}\right)$ to $K e y^{\text {List }}$.

(b) RKExtract $\left(I D_{i}, \mathcal{T}, I D_{j}\right): \mathcal{B}$ first searches whether there is a tuple $\left(*, I D_{i}, \mathcal{T}, I D_{j}\right.$, $\left.r k_{\mathcal{T} \mid I D_{i} \rightarrow I D_{j}}, \theta_{1}, \theta_{2}, *\right)$ in $R e K e y^{\text {List }}$. If yes, $\mathcal{B}$ returns $r k_{\mathcal{T} \mid I D_{i} \rightarrow I D_{j}}$ as the result, where $*$ is the wildcard. Otherwise, $\mathcal{B}$ proceeds as follows:

- If $\left(1, I D_{i}, s k_{I D_{i}}\right)$ exists in $K e y^{L i s t}, \mathcal{B}$ uses $s k_{I D_{i}}$ to generater the re-encryption key $r k_{\mathcal{T} \mid I D_{i} \rightarrow I D_{j}}$ via algorithm RKeyGen as in the real scheme. Returns the re-encryption key to $\mathcal{A}$ and adds $\left(*, I D_{i}, I D_{j}\right.$, $\left.r k_{\mathcal{T} \mid I D_{i} \rightarrow I D_{j}}, \theta_{1}, \theta_{2}, 1\right)$ to $R e K e y^{\text {List }}$, where $\theta_{1} \in\{0,1\}^{k_{1}}$, $\theta_{2} \in\{0,1\}^{k_{2}}$ are randomly chosen in the RKeyGen algorithm.

- Otherwise, $\mathcal{B}$ flips a biased coin $\beta$. If $\beta=1, \mathcal{B}$ queries the $\operatorname{Extract}\left(I D_{i}\right)$ oracle to get $s k_{I D_{i}}$, and then generates $r k_{\mathcal{T} \mid I D_{i} \rightarrow I D_{j}}$ via algorithm $R K$ eyGen as in the real scheme. Returns the re-encryption key to $\mathcal{A}$ and adds $\left(1, I D_{i}, s k_{I D_{i}}\right)$ and $\left(*, I D_{i}, I D_{j}, r k_{\mathcal{T} \mid I D_{i} \rightarrow I D_{j}}, \theta_{1}, \theta_{2}, 1\right)$ to $K e y^{\text {List }}$ and ReKey ${ }^{\text {List }}$ respectively, where $\theta_{1} \in\{0,1\}^{k_{1}}, \theta_{2} \in\{0,1\}^{k_{2}}$ are randomly chosen in the RKeyGen algorithm. If $\beta=0, \mathcal{B}$ randomly chooses $\theta_{1} \in\{0,1\}^{k_{1}}, \theta_{2} \in$ $\{0,1\}^{k_{2}}, s_{x} \in Z_{p}^{*}$ and computer $a, b$ as in the real scheme. Then $\mathcal{B}$ sets $r k_{1}=\phi_{1}, r k_{2}=$ $\phi_{2}, r k_{3}=\phi_{3}$ for randomly chosen $\phi_{1}, \phi_{2}, \phi_{3} \in G$. Then $\mathcal{B}$ constructs $r k_{3}, r k_{4}, r k_{5}, r k_{6}, r k_{7}, s v k^{\prime}, S^{\prime}$ to encrypted a random $\left(\theta_{1} \| \theta_{2}\right) \in\{0,1\}^{l_{1}}$ as in the real scheme. $\mathcal{B}$ forwards the reencryption key to $\mathcal{A}$ and adds $\left(*, I D_{i}, \mathcal{T}, I D_{j}, r k_{\mathcal{T} \mid I D_{i} \rightarrow I D_{j}}, \theta_{1}, \theta_{2}, 0\right)$ to ReKey ${ }^{\text {List }}$.

(c) $\operatorname{Dec} 1\left(I D_{j}, C_{I D_{j}}^{(1)}\right): \mathcal{B}$ first verifies equations (1)-(4) and (6)-(8). If the equations do not hold, output $\perp$ and abort. Otherwise, $\mathcal{B}$ proceeds:

- If $\left(i, I D_{j}, s k_{I D_{j}}\right)$ exists in Key ${ }^{\text {List }}, \mathcal{B}$ recovers $m$ using $s k_{I D_{j}}$.

- Otherwise, $\mathcal{B}$ issues $\left(s v k^{\prime}, r k_{3}, r k_{4}, r k_{5}, r k_{6}, r k_{7}, S^{\prime}\right)$ to the decryption oracle of $\mathrm{mWIBE}$ and get $\theta_{1}, \theta_{2}$. Then using $\theta_{1}, \theta_{2}, \mathcal{B}$ can recover $m$ as in the real scheme.

3. Challenge: Once $\mathcal{A}$ decides that Phase 1 is over, it outputs two equal length message $\left(m_{0}, m_{1}\right)$ and a target identity $I D^{*}$. If $\left(1, I D^{*}, s k_{I D^{*}}\right)$ exists in the $K e y^{\text {List }}, \mathcal{B}$ outputs random bits and aborts. Else, $\mathcal{B}$ choose an identity $I D_{i} \neq I D^{*}$, and forwards $\left(m_{0}, m_{1}, I D_{i}, W^{*}\right)$ to the challenge oracle of mWIBE to obtain the result $C_{I D_{i}}^{(2)} \cdot \mathcal{B}$ then issues $R K \operatorname{Extract}\left(I D_{i}, \mathcal{T}, I D^{*}\right)$, where $\mathcal{T}\left(W^{*}\right)=1$ to get $r k_{\mathcal{T} \mid I D_{i} \rightarrow I D^{*}} . \mathcal{B}$ uses $r k_{\mathcal{T} \mid I D_{i} \rightarrow I D^{*}}$ to re-encrypt $C_{I D_{i}}^{(2)}$ as the real scheme and get the challenge ciphertext $C_{I D^{*}}^{(1) *}$. Finally, $\mathcal{B}$ sends the challenge ciphertext $C_{I D^{*}}^{(1) *}$ to $\mathcal{A}$.

4. Query phase 2: A continues making queries as in the query phase 1 with the restrictions described in the IND-level1-CCA game.

5. Guess: When A outputs a guess $b^{\prime} \in\{0,1\}, \mathcal{B}$ outputs $b^{\prime}$.

Probability analysis. If $\mathcal{B}$ does not abort, $\mathcal{A}$ 's view is identical to the real scheme. We define Abort be the event of $\mathcal{B}^{\prime} s$ aborting during the simulation of Extract query. Let $q_{e}$ denote the 
total number of Extract queries, we have $\operatorname{Pr}[\neg$ Abort $] \geq \delta^{q_{e}}(1-\delta)$, which is maximized at $\delta_{\text {opt }}=\frac{q_{e}}{\left(1+q_{e}\right)}$. Using $\delta_{\text {opt }}$, the probability $\operatorname{Pr}[\neg A b o r t]$ is at least $\frac{1}{\dot{e}\left(1+q_{e}\right)}$, where $\dot{e}$ is the base of the nature logarithm. Therefor, we have

$$
\varepsilon^{\prime} \geq \frac{\varepsilon}{\dot{e}\left(1+q_{e}\right)}-A d v_{H_{1}, \mathcal{A}}^{T C R}-A d v_{H_{2}, \mathcal{A}}^{T C R}-A d v_{F, \mathcal{F}}^{P R F}
$$

This completes the proof of Theorem 3. 